\title{
One third of British oral surgeons selectively recall patients following third molar surgery.
}

\author{
Pratt C A, Hekmat M, Pratt S D, Zaki G A, Barnard J D W. Controversies in thirds molar surgery - the \\ national view on review strategies. Br J Oral MaxFac Surg. 1997; 35: 319-322.
}

Objectives To ascertain practice relating to review following third molar surgery

Method A questionnaire survey of Fellows of the British Association of Oral and Maxillofacial Surgeons.

Results There was an $86 \%$ response rate to the questionnaire, a small number of uncompleted questionnaires left $84.7 \%$ for analysis. While $62 \%$ of fellows routinely reviewed their patients $31 \%$ did not, calling only those patients for review who reported a neurological deficit or those with associated pathology. In 1992 the proportion of those requesting a regular review was $85.7 \%$.

Conclusions The paper does not have any specific conclusions but it does discuss a number of relevant issues and suggests a number of safeguards for those pursuing a policy of selective recall.

Address for reprints: C A Pratt, Maxillofacial Unit, Queen Alexandra Hospital, Cosham, Portsmouth,UK

\section{Commentary}

The study by Pratt et al summarizes the results of a survey of Fellows of the British Society of Oral and Maxillofacial Surgery. The fellows were asked whether they routinely or selectively scheduled patients for postoperative visits after $3 \mathrm{rd}$ molar removal. While a majority of surgeons do schedule routine appointments (62\%), clearly a significant number of surgeons utilize a selective postoperative follow up regimen. A minority opinion $(2 \%)$ felt it was malpractice to not schedule routine postoperative visits.

The response rate to the written questionnaire $(86 \%)$ is very good. As such the survey reports the opinions of a majority of fellows in the specialty. Given the unlikely scenario of having the non responders universally accept or reject a choice for postoperative follow up does not alter the study's conclusions.

Having read this article when it was published and asked the question "What are the advantages (and disadvantages) of a routine postoperative visit policy?' I changed my own practice pattern from routine to selective postoperative visits using the safeguards outlined in the article. Patients anticipated to be at high risk for postoperative complications are routinely scheduled for postoperative visits. Patients with an anticipated low risk for complications are given an appointment for follow up, but asked to call and cancel the appointment if they have no problems. Implementation of this practice led to a modest reduction (20\%) in postoperative visits with no apparent change in the postoperative complication rates or patient satisfaction.

The major advantage of a selective postoperative visit routine is time savings for both practitioner and patient. While the encounter is brief for the practitioner, the sheer volume of postoperative visits can be unwieldy. The patient avoids lengthy time commitments for a brief and likely unproductive office visit. It is not unusual for a patient to commit one-half to one day (travel, parking, waiting room time) for a five to ten minute encounter with the clinician.

As a clinician, it is satisfying to see patients preoperatively, intraoperatively, and then 'close the loop' with a postoperative visit. Because patients, not clinicians, bear a disproportionate amount of the time commitment for an office visit, I do not feel compelled to see every third molar extraction case postoperatively. Sharing decision making for postoperative visits with a responsible adult patient has validity in most cases.

\author{
Thomas Dodson \\ Oral and Maxillofacial Surgery \\ Massachusetts General Hospital, \\ Boston, MA 02114 \\ USA
}

